\title{
Caracterización estructural de la cascarilla de arroz modificada como adsorbente alternativo y eficiente para la remoción de $\mathrm{Cr}(\mathrm{VI})$ en solución \\ Structural characterization of the husks from modified rice as an alternative absorbant and effective for the removal of $\mathrm{Cr}(\mathrm{VI})$ in solution Caracterização estrutural de cascas de arroz
modificados como adsorvente alternativo e eficiente
para a remoção do $\mathrm{Cr}(\mathrm{VI})$ em solução
}

\author{
Gloria María Doria Herrera1, Angelina Hormaza Anaguano² \& Darío Gallego Suarez ${ }^{3}$ \\ ${ }^{1}$ Químico, Magister en Ciencias- Química. ²Químico, Magister en Ciencias- Química Orgánica, \\ Doctor Rerum Naturualien, Doctora en Ciencias Químicas. \\ ${ }^{3}$ Ingeniero Químico, Magister en Ingeniería Ambiental \\ 'Escuela de Ciencias Básicas, Tecnologías e Ingenierías. Universidad Nacional Abierta y a Distancia

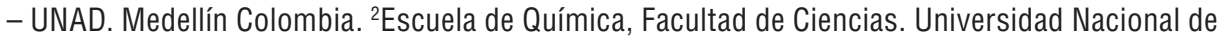 \\ Colombia - Sede Medellín. Medellín, Antioquia, Colombia. ${ }^{3}$ Escuela de procesos. Facultad de Minas. \\ Universidad Nacional de Colombia - Sede Medellín. Medellín, Antioquia, Colombia \\ 1gloria.doria@unad.edu.co, ${ }^{2}$ ahormaza@unal.edu.co, ${ }^{3}$ dgallego@unal.edu.co
}

\section{Resumen}

Este artículo describe la caracterización estructural de la cascarilla de arroz modificada mediante un proceso de oxidación avanzada con el reactivo Fenton, dicha transformación fue llevada a cabo con el propósito de incrementar el número de sitios activos sobre la superficie de este material y generar por tanto, un adsorbente con mayor capacidad de remoción de los iones $\mathrm{Cr}$ (VI) en solución, en comparación con la cascarilla de arroz natural. La caracterización del material modificado fue realizada mediante distintas técnicas experimentales que incluyeron, el análisis de los grupos funcionales presentes en la superficie del adsorbente a través de la espectroscopia de infrarrojo con reflectancia difusa, (DRIFT), así mismo, un análisis de la morfología de la superficie de la cascarilla mediante microscopia electrónica de barrido, (SEM), un análisis bromatológico y termogravimétrico, (TGA). Todas estas evaluaciones fueron llevadas a cabo sobre el adsorbente previo y posterior a su proceso de modificación. Los resultados de DRIFT señalan un ligero desplazamiento y una atenuación en las frecuencias de vibración de los grupos funcionales implicados en la modificación estructural, en tanto que el análisis SEM indicó cambios apreciables en la morfología del material adsorbente. Por su parte, el análisis bromatológico mostró modificaciones considerables en los porcentajes de los componentes del material, cuya presencia fue ratificada mediante el análisis TGA, al alcanzar su descomposición a las temperaturas esperadas. En definitiva, todos los resultados confirman la modificación estructural del adsorbente evaluado. 
Palabras clave: adsorción, composición, infrarrojo, morfología, técnicas espectroscópicas.

\section{Abstract}

This article describes the structural characterization of the husk of modified rice through the process of advanced oxidation with the reagent Fenton; the transformation was carried out with the intention of increasing the number of active sites on the material's surface, and consequently to generate an absorbent with a greater capacity for the removal of $\mathrm{Cr}(\mathrm{VI})$ ions in solution, compared to the husk of natural rice. The characterization of modified material was determined through distinct experimental techniques that include the analysis of the functional groups present on the absorbent's surface through infrared spectroscopy with diffuse reflectance, (DRIFT), likewise, a surface morphology analysis of the husk through a scanning electron microscope (SEM), a compositional analysis and thermogavimetric (TGA). All of these tests were carried out before and after the modification process. The results of the DRIFT point towards a slight shift and attenuation in the vibration frequencies of the functional groups implicated in the structural modification, whereas the SEM analysis indicated noticeable changes in the morphology of the absorbent material. Meanwhile, the compositional analysis showed considerable changes in the percentage of the material components, whose presence was confirmed through the TGA analysis, after decomposing at the expected temperatures. In short, all results confirm the structural modification of the absorbent tested.

Key-words: adsorption, composition, infrared, morphology, spectroscopic techniques.

\section{Resumo}

Este artigo descreve a caracterização estrutural da casca de arroz modificado pelo processo de oxidação avançada com o reagente de Fenton, esta transformação foi levada a cabo com a finalidade de aumentar o número de locais ativos na superfície deste material e, portanto, gerar maior capacidade adsorvente para remoção de íons de $\mathrm{Cr}$ (VI) em solução, em comparação com a casca de arroz natural. A caracterização do material modificado foi realizada por várias técnicas experimentais que envolvem a análise dos grupos funcionais presentes na superfície do adsorvente através da espectroscopia de infravermelhos de refletância difusa (DRIFT), do mesmo modo, uma análise da morfologia da superfície da casca por microscopia eletrônica de varredura (MEV), uma análise bromatológica e termogravimétrica (TGA). Todas estas avaliações foram realizadas sob o adsorvente antes e depois do processo de modificação. Os resultados do DRIFT indicam uma ligeira mudança e atenuação nas frequências de vibração dos grupos funcionais envolvidos na modificação estrutural, enquanto que a análise de MEV indicou alterações significativas na morfologia do material adsorvente. Enquanto isso, a análise bromatológica mostrou mudanças significativas nas percentagens dos componentes do material, cuja presença foi confirmada por análise TGA, atingindo a sua decomposição a temperaturas esperadas. Em suma, todos os resultados confirmam a modificação estrutural do adsorvente avaliado.

Palavras-chave: adsorção, composição, infravermelho, morfologia, técnicas espectroscópicas. 
Introducción

La industria de curtido de cueros es sin duda uno de renglones más representativos en la economía del departamento de Antioquia, Colombia, con un número aproximado de siete curtiembres en el área metropolitana de la ciudad de Medellín y sus alrededores: cinco ubicadas en Medellín, una en Guarne y una en Sonsón (Alzate Tejada, Aragón Guzmán, \& Tobón, 2004). La descarga de estos efluentes al ambiente con un mínimo o nulo tratamiento previo ha generado un incremento alarmante en la concentración de metales pesados, particularmente de $\mathrm{Cr}(\mathrm{VI})$, elemento que debido a su alta toxicidad y elevado potencial cancerígeno puede provocar graves problemas de salud en los seres vivos, así como repercuciones nefastas para el medio circundante (ATSDR; 2004). La Agencia de Protección Ambiental de los Estados Unidos, EPA, ha clasificado el cromo como contaminante de prioridad 1, debido a su estabilidad en solución acuosa y por tanto, alta movilidad en diferentes ambientes.

Dentro de los métodos de tratamiento de contaminantes en solución, la adsorción ha sido señalada como una alternativa innovadora para la desintoxicación y recuperación de este tipo de efluentes; ventajas como su eficiencia, facilidad del proceso y retención de la molécula completa del contaminante, han estimulado múltiples investigaciones enfocadas a evaluar adsorbentes novedosos y de bajo costo (Singh, Hasan, Talat, Singh, \& Gangwar, 2009; Kishore, Krishani, Chiristodoulatos, \& Bobdu, 2008), dado que el carbón activado, pese a su notable capacidad de retención de una amplia variedad de metales disueltos, demanda procesos económicamente elevados debido a los altos costos de manufacturación y regeneramiento (Rodríguez- Reinoso, 1997; Abdel- Nasser, El-Hendawy, Samra, \& Girgis, 2001; Mohamed \& Mohamed, 2004; Bello, Cid, \& García y Arraigada, 2002; Juang, Tseng, \& Wu, 2001).
Así, un vasto espectro de residuos agrícolas ha sido valorado para el tratamiento tanto de metales como de colorantes en solución con resultados satisfactorios (Doria, Hormaza, \& Gallego, 2011; Hormaza, Figueroa, \& Moreno, 2012; Gupta \& Suhas, 2009; Crini, 2006). No obstante, para la remoción de metales y en particular de $\mathrm{Cr}(\mathrm{VI})$, la utilización de estos subproductos de forma natural ha conducido en muchos casos a la obtención de porcentajes de retención relativamente bajos, cercanos al 71\% (Manjeet, Garg, Singh, \& Garg, 2009). Por esta razón, y con el propósito de incrementar la capacidad de adsorción, investigaciones recientes se han enfocado a la modificación estructural de estos materiales a través de diferentes tratamientos tanto físicos como químicos.

Entre estos estudios cabe señalar el desarrollado por Wang y sus colegas (Wang \& Hanafiah, 2008), quienes presentaron el uso de cascarilla de arroz, cáscaras de maní, aserrín y cáscara de banano, entre otros residuos, para la remoción de metales pesados tales como cadmio, cobre, plomo, zinc y níquel usando ácidos orgánicos, bases, agentes oxidantes y compuestos orgánicos, obteniendo capacidades de adsorción satisfactorias. Por su parte, Kumar y colegas (Kumar \& Bandyopadhyay, 2006), realizaron el pretratamiento con hidróxido de sodio, bicarbonato de sodio, ácido clorhídrico y epiclorhidrina sobre la cascarilla de arroz para aumentar la adsorción de iones de Cd (II); Marshall y colegas (Marshall, Wartelle, Boler, Johns, \& Toles, 1999) evaluaron la soya pretratada con ácido cítrico en medio básico para aumentar la eficiencia de remoción de Cu (II) en solución. Así mismo, Wong y colaboradores (Wong, Low, \& Haron, 2003) utilizaron cascarilla de arroz modificada con ácidos policarboxílicos, (ácido cítrico, salicílico, oxálico, mandélico, málico y tartárico) para mejorar la remoción de los iones $\mathrm{Cu}$ (II) y $\mathrm{Pb}$ (II) en solución; Mehmet y su equipo de trabajo (Mehmet, Argun, \& Dursun, 2008); 
llevó a cabo la modificación de aserrín de madera con el reactivo Fenton para incrementar los sitios activos sobre la superficie del adsorbente, lo cual permitió la eliminación de Cd (II) en solución con porcentajes cercanos al $97 \%$.

Por otro lado, la cascarilla de arroz es un subproducto agrícola de amplia abundancia en nuestro país; se estima que anualmente se generan $2.145 .100 \mathrm{t}$ de arroz, que dan lugar a $578.452 \mathrm{t}$ de desechos, de los cuales solo un tercio es aprovechado en distintos fines, permaneciendo una gran cantidad como material de desecho (FEDEARROZ, 2006).

En la perspectiva de generar adsorbentes de bajo costo, fácil disponibilidad y con alta capacidad de remoción para alcanzar mayor eficiencia en la implementación de tecnologías limpias como es el proceso de adsorción, se llevó a cabo la modificación estructural de la cascarilla de arroz mediante el proceso de oxidación avanzada con el reactivo Fenton. En este trabajo se presenta su completa caracterización estructural que incluye un análisis espectroscópico, bromatológico y termogravimétrico.

\section{Materiales y métodos}

\section{Caracterización y preparación del material adsorbente}

La cascarilla de arroz fue adquirida de las áreas de cultivo de arroz del departamento de Córdoba (Colombia). Este producto se fragmentó y se lavó repetidamente (mínimo cuatro veces) con agua destilada para eliminar coloraciones alternas que interfieren con las respectivas mediciones. Posteriormente, el material se sometió a secado durante 48 horas en un horno a $90^{\circ} \mathrm{C}$. El sólido seco fue molido y tamizado para obtener un tamaño de partícula homogéneo (> $500 \mu \mathrm{m}$ ) (Tewari, Vasudevan, \& Guha, 2005); (APHA -AWWA, 1999).

\section{Preparación del material modificado}

Para la preparación de la cascarilla de arroz modificada se utilizó el reactivo Fenton, cuya relación óptima de $\left[\mathrm{Fe}^{+}\right] /\left[\mathrm{H}_{2} \mathrm{O}_{2}\right]$ se determinó mediante la variación de la velocidad a 0.001 controlando la temperatura y el $\mathrm{pH}$ (Mehmet, Sukru, Mustafa, \& Metin, 2008). Para controlar el valor del $\mathrm{pH}$ de las muestras se preparó un tampón $\mathrm{KHC}_{6} \mathrm{H}_{4}(\mathrm{COO})_{2}$ I $\mathrm{HCl}$ a $\mathrm{pH}=3.0$

El procedimiento de modificación del adsorbente se llevó a cabo de la siguiente manera: $2.5 \mathrm{~g}$ de cáscara de arroz fueron depositados en un matraz Erlenmeyer que contenía $25 \mathrm{~mL}$ de reactivo Fenton. Las mezclas se agitaron a 127 rpm durante todo el experimento de la modificación, a continuación, las muestras se filtraron y se lavaron con agua destilada/desionizada cinco veces y luego el sustrato procesado se sometió a $85^{\circ} \mathrm{C}$ durante 24 horas, y luego a secado en bolsas de plástico. Este material modificado se utilizó para realizar las pruebas requeridas para la eliminación de $\mathrm{Cr}(\mathrm{VI})$, donde la capacidad máxima de adsorción correspondió a la muestra con el mayor peso de metal adsorbido. Con la mejor proporción de reactivo de Fentón, se evaluó la influencia de otros parámetros tales como pH, concentración de metal, dosificación de adsorbente y tiempo de contacto.

\section{Caracterización del material adsorbente}

\section{Análisis bromatológico}

Este análisis se realizó en el Laboratorio de Análisis Químico y Bromatológico de la Universidad Nacional de Colombia - Sede Medellín. En particular, se llevaron a cabo determinaciones de fibra detergente ácido (FDA), fibra detergente neutro (FDN) y lignina antes y después de la modificación, siguiendo el método Van Soest (Valverde, Sarria, \& Monteagudo, 2007). 
Análisis de los Grupos Funcionales - DRIFT

Para verificar la variación en las frecuencias de vibración de los grupos funcionales presentes en la superficie de la cascarilla de arroz modificada, se tomaron los espectros de DRIFT de la muestra previa y posterior al proceso de modificación. Las pastillas fueron preparadas con bromuro de potasio $(\mathrm{KBr})$ y medidas en un espectrofotómetro marca Termo-Nicolet Avatar 330 en la Sede de Investigaciones Universitarias, SIU, de la Universidad de Antioquia.

\section{Morfología superficial - SEM}

Micrografías de microscopía electrónica de barrido (SEM) del material de estudio fueron tomadas antes y después de la adsorción de metal. Este análisis se llevó a cabo con el microscopio electrónico de Barrido JEOL-JSM 5910LV del Laboratorio de Microscopía Avanzada de la Universidad Nacional de Colombia-Sede Medellín

\section{Análisis termogravimétrico - TGA}

En el análisis termogravimétrico se registró la masa de las muestras de forma lineal desde la temperatura ambiente hasta temperaturas del orden de $1200{ }^{\circ} \mathrm{C}$.
El termograma registrado se analizó antes y después de la modificación. Estas determinaciones se realizaron en el Analizador Termogravimétrico (TGA) marca Q500 TA Instrument en la Sede de Investigaciones Universitarias, SIU, de la Universidad de Antioquia.

\section{Resultados y discusión Análisis bromatológico}

Para conocer la composición del material adsorbente se llevó a cabo un análisis de la composición de la cascarilla de arroz natural y del material modificado (Tabla 1). Estos resultados demostraron que el material modificado tiene porcentajes más altos que la cascarilla natural, lo que permite suponer que su proceso de modificación condujo a la oxidación de los taninos, aumentando de forma apreciable los porcentajes de lignina, celulosa y hemicelulosa.

Es importante resaltar que el porcentaje de lignina, del orden de $6.66 \%$, es sorprendentemente menor que el reportado en otras investigaciones (Manjeet, Garg, Singh, \& Garg, 2009). Además, otros autores atribuyen a la lignina un predominante papel en la adsorción de metales ya que posee mayor velocidad que la celulosa y hemicelulosa para adherirse a los metales (Mehmet, Argun, \& Dursun, 2008).

Tabla 1. Análisis composicional de la cascarilla de arroz natural y modificada (\% en base seca)

\begin{tabular}{lcccccc}
\hline \multicolumn{1}{c}{ Material } & FDN & FDA & Lignina & ${ }^{*}$ Celulosa & ${ }^{*}$ Hemicelulosa & Cenizas \\
\hline $\begin{array}{l}\text { Cascarilla de } \\
\text { arroz natural }\end{array}$ & 77.97 & 66.78 & 6.66 & 60.12 & 11.19 & 15.90 \\
$\begin{array}{l}\text { Cascarilla de } \\
\text { arroz modificada }\end{array}$ & 90.66 & 75.26 & 7.26 & 68.00 & 15.40 & 9.31 \\
\hline
\end{tabular}

* Valores calculados. Para el cálculo se tomó como principio la fracción de FDN (lignina + celulosa + hemicelulosa) y la fracción de FDA (lignina + celulosa). Análisis realizado en el Laboratorio de Análisis Químico y Bromatológico de la Universidad Nacional de Colombia-Medellín. 


\section{Análisis de espectroscopia infrarroja}

Un análisis de espectroscopia infrarroja de reflectancia difusa (DRIFT) fue realizado para la muestra de cascarilla de arroz natural (Figura 1) y cascarilla de arroz modificada (Figura 2).

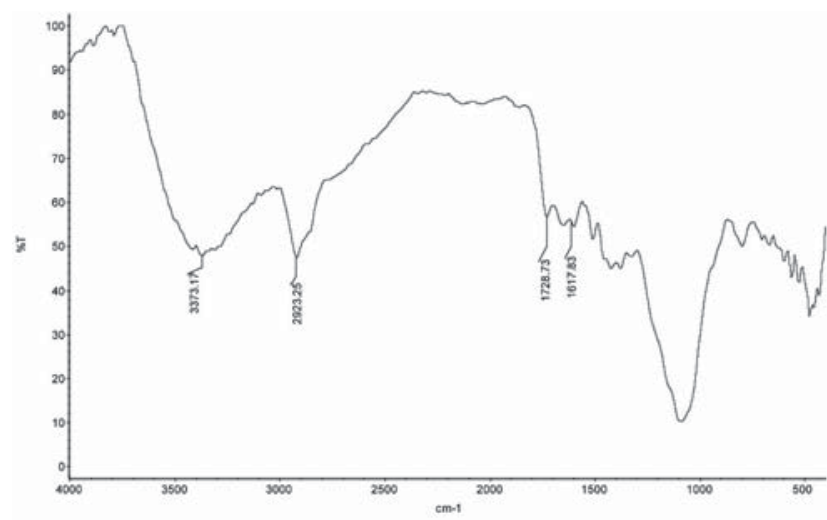

Figura 1. Espectro infrarrojo de la cascarilla natural

En la (figura 1) cabe resaltar como bandas principales, la vibración alrededor de $3370 \mathrm{~cm}^{-}$correspondiente al enlace $\mathrm{O}-\mathrm{H}$ asociado, para el enlace $\mathrm{C}-\mathrm{H}$ alifático las típicas señales entre $2.900-3.000 \mathrm{~cm}$, las vibraciones asociadas al enlace $\mathrm{C}=\mathrm{C}$ anillo aromático se registran entre 1490 y $1617 \mathrm{~cm}$; finalmente las bandas del enlace C-O alrededor de $1000 \mathrm{~cm}^{-}$y una banda en 1728 que indica la presencia del grupo $\mathrm{C}=\mathrm{O}$. El espectro de la cascarilla modificada, (figura 2), muestra una banda característica ubicada aproximadamente en $1730 \mathrm{~cm}$; atribuible al grupo carbonilo del ácido carboxílico(COOH), así como la banda de vibración típica del enlace $\mathrm{O}-\mathrm{H}$ asociado alrededor de $3400 \mathrm{~cm}^{-}$, pero con una menor intensidad en relación a dicha banda registrada en la cascarilla natural. En general, se observó una notable disminución de las bandas de vibración en el adsorbente modificado.

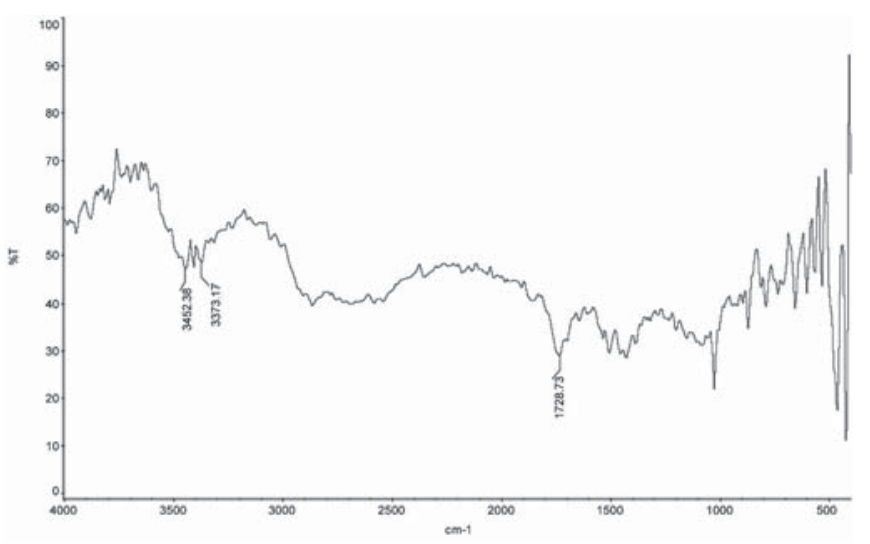

Figura 2. Espectro infrarrojo de la cascarilla modificada

\section{Micrografía electrónica de barrido (SEM)}

La micrografía electrónica de barrido se utilizó para analizar la superficie de la cáscara de arroz antes y después de la modificación (figura 3). Es importante mencionar que a nivel morfológico se observa en la superficie del material una notable descamación como consecuencia de la reacción de modificación sin alteración del tamaño de poro, resultados que apoyan la ocurrencia de la transformación química deseada. También se realizó un microanálisis (EDX) para determinar con base en la abundancia de sus componentes, cuales son los principales compuestos presentes, la cáscara de arroz natural mostró altos niveles de óxidos de silicio, carbonato de calcio, rastros de óxido de magnesio, de potasio y aluminio, como se desprende del análisis de la composición química correspondiente, información acorde con lo reportado, (figura 4) (Valverde, Sarria, \& Monteagudo, 2007). 


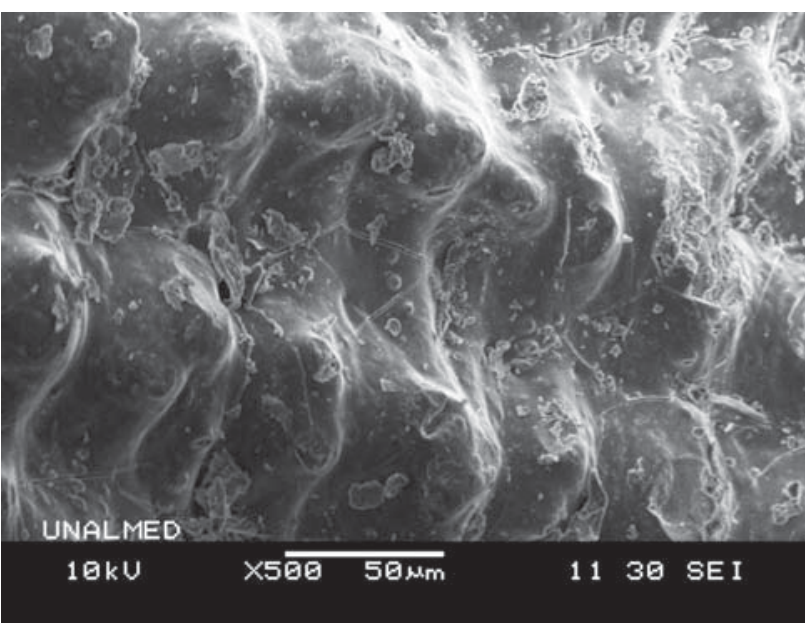

Figura 3. Microscopia electrónica de barrido de la cascarilla natural.

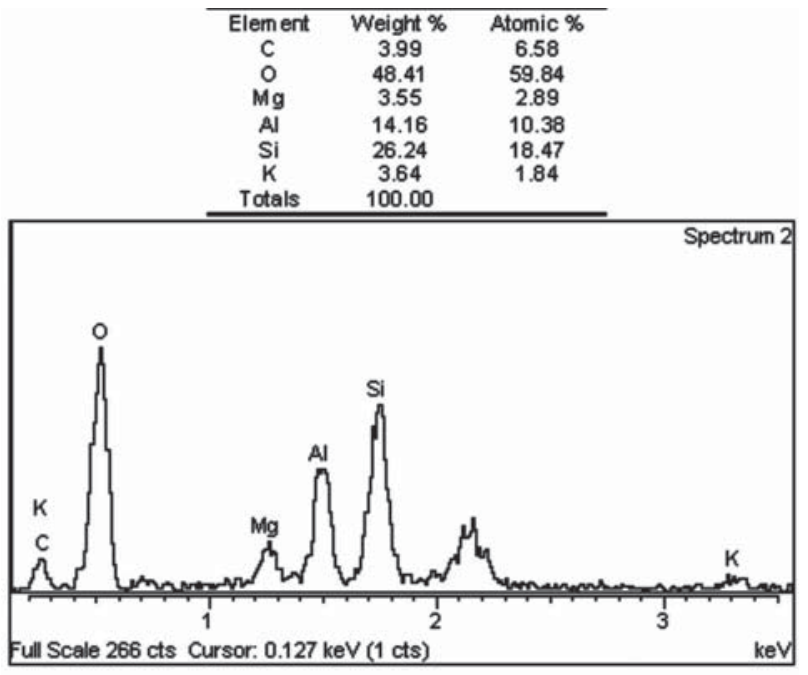

a. Cascarilla natural

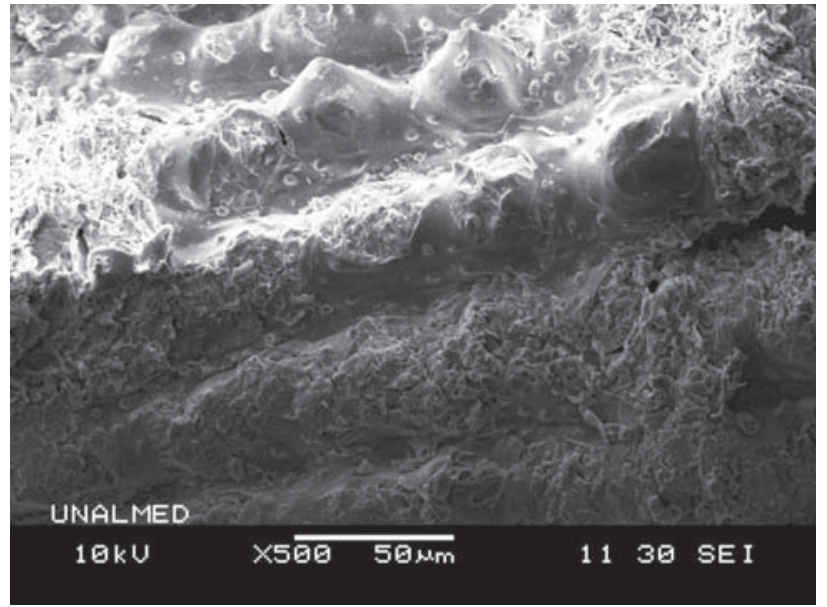

Figura 4. Microscopia electrónica de barrido de la cascarilla modificada.

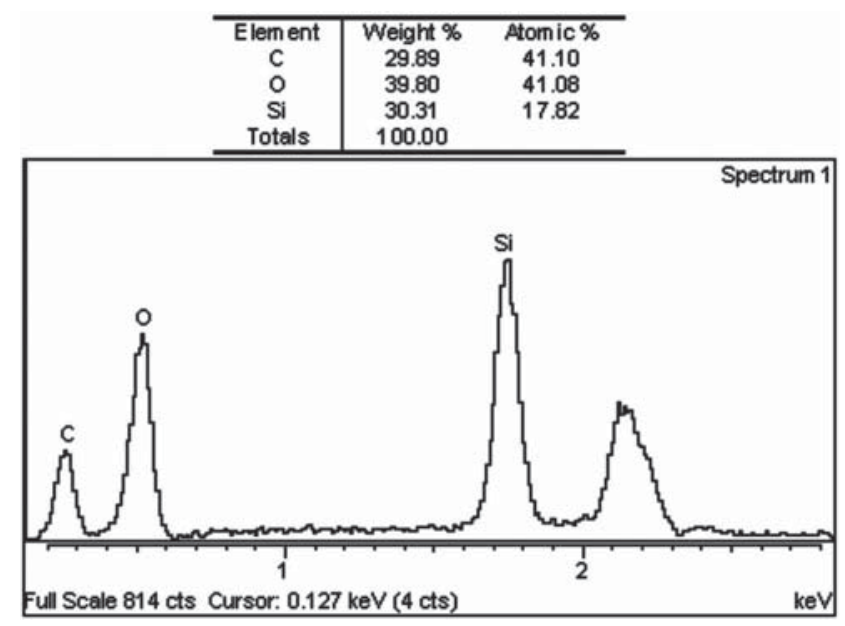

b. Modificada con Fenton $\left[\mathrm{Fe}^{+}\right] /\left[\mathrm{H}_{2} \mathrm{O}_{2}\right]=0,001$

Figura 5. Microanálisis realizado en el software INCA- SEM para la cascarilla de arroz natural y cascarilla modificada.

\section{El análisis termogravimétrico (TGA)}

El análisis termogravimétrico permite determinar la presencia de un determinado material mediante la descomposición de sus moléculas más pequeñas con ayuda de incrementos progresivos de temperatura y de este modo, identificar ciertos tipos de estructuras. Los datos obtenidos a partir del termograma para el adsorbente de estudio son mostrados en las figuras 6 y 7.

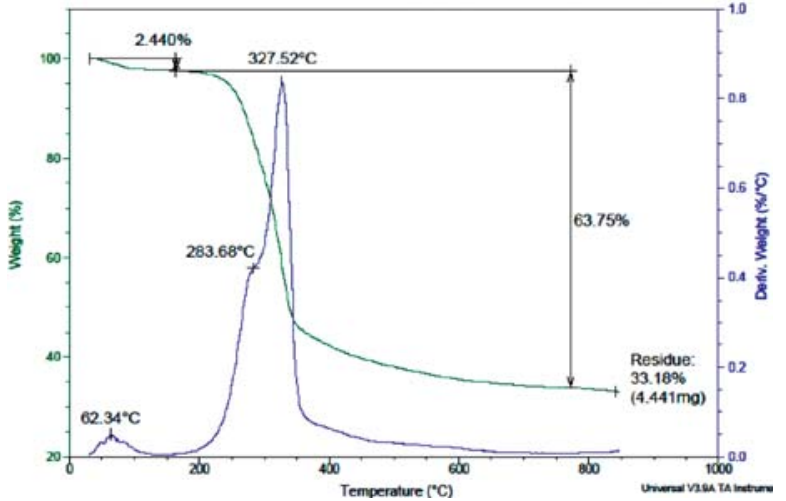

Figura 6. Análisis termogravimétrico de la cascarilla natural. 


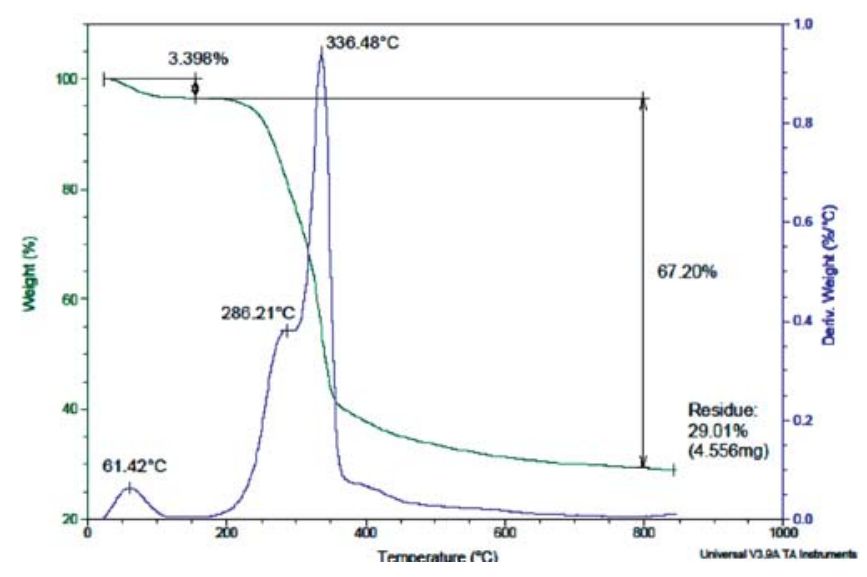

Figura 7. Análisis termogravimétrico de la cascarilla modificada.

Este experimento se llevó a cabo bajo atmósfera de nitrógeno para evitar la oxidación de los compuestos y con ello asegurar la exactitud de los resultados. Las derivaciones obtenidas son muy coherentes con lo reportado (Yang, Yang, Lee, \& Zheng, 2007). Inicialmente, el intervalo de temperatura que va desde 0 a $100{ }^{\circ} \mathrm{C}$, muestra que a una temperatura máxima de $62.34 \stackrel{\circ}{\circ} \mathrm{C}$ ocurrió una pérdida de peso del $2.44 \%$ para la cascarilla natural (figura 6) y a $61.42{ }^{\circ} \mathrm{C}$ una pérdida de peso del $3.39 \%$ para el material modificado (figura 7 ), estas pérdidas se atribuyen a la humedad de la muestra. Luego se inició la fácil descomposición de la hemicelulosa, entre $220-315^{\circ} \mathrm{C}$, con temperatura máxima de $283.68{ }^{\circ} \mathrm{C}$ para la cascarilla natural y $286.21 \stackrel{\circ}{C}$ para la cascarilla modificada; inmediatamente con el inicio de la pirólisis de la celulosa entre 315-400 드, se observó su descomposición a una temperatura máxima de $327.52{ }^{\circ} \mathrm{C}$ para la cascarilla natural y de $336.48{ }^{\circ} \mathrm{C}$ para la modificada. Finalmente, se conoce que la lignina se descompone secuencialmente en el intervalo de temperatura ambiente hasta $900{ }^{\circ} \mathrm{C}$, por ello y de acuerdo a la literatura, no se registró una banda específica para su descomposición a una temperatura determinada.

\section{Conclusiones}

La caracterización de adsorbente natural y modificado de la cascarilla de arroz, se logró mediante técnicas de espectroscopia de infrarrojo con reflectancia difusa, (DRIFT), microscopia electrónica de barrido, (SEM), análisis bromatológico y termogravimétrico, (TGA).

Los análisis confirman la ocurrencia de la modificación estructural efectuada sobre la superficie del adsorbente de estudio. El análisis DRIFT mostró una notable atenuación en la intensidad de las bandas de vibración de los principales grupos funcionales para el material modificado. La micrografía electrónica de barrido (SEM) reveló cambios significativos en el material modificado, en particular, una apreciable descamación y señaló que la transformación ocurrió a nivel estructural, pues el tamaño de poro se mantuvo invariable. El análisis termogravimétrico permitió evidenciar que los principales componentes de la cascarilla natural y cascarilla modificada de arroz son celulosa, hemicelulosa, lignina y ceniza, cuya composición se confirmó mediante el análisis bromatológico, el cual arrojó porcentajes más altos para cada uno de los componentes en el material modificado, sugiriendo que el proceso de modificación efectivamente dio lugar a la oxidación de los taninos presentes en la superficie del adsorbente.

\section{Agradecimientos}

Los autores extienden sus agradecimientos a la Universidad Nacional de Colombia - Sede Medelín por el apoyo a través de la infraestructura del Laboratorio de Química Experimental, así como a la Dirección de Investigación de la Sede Medellín, DIME, por el financiamiento del proyecto Código 201010011038 y por el apoyo financiero a través del Programa de Jóvenes Investigadores e Innovadores de Colciencias 2009. 


\section{Literatura citada}

1. Abdel- Nasser, A El-Hendawy; Samra, S.E. \& Girgis, B.S. (2001). Adsorption characteristics of activated carbons obtained from corncobs. Colloids and Surface A: Physicochemical and Engineering Aspects, 180: 209-221.

2. Alzate A., Aragon M. \& Tobon, O. (2004). Proyecto gestion ambiental en la industria de curtiembres en Colombia. Antioquia: Centro de Produccion más limpia y Gestión ambiental.

3. APHA -AWWA. (1999). Standar Methods: Examination of Waste and Wastewater. Software 20th.

4. Argun, M.E. \& Dursun, S. (2008). A new approach to modification of natural adsorbent for heavy metals adsorption. Bioresource Technology, 99 (7): 2516-2527.

5. Argun, M.E. Sukru, D., Mustafa, K. \& Metin, G. (2008). Activation of pine cone using Fenton oxidation for $\mathrm{Cd}(\mathrm{II})$ and $\mathrm{Pb}(\mathrm{II})$ removal. Biresource Thecnology, 99 (18): 8691-8698.

6. ATSDR (2004). Agencia para sustancias tóxicas y el registro de enfermedades. Reseña toxicológica del cromo (versión para comentario público) (en inglés). EEUU.

7. ATSDR;Agencia para Sustancias Toxicas y el registro de enfermedades (2008). Reseña toxicológica del cromo (versión para comentario público) (en inglés). Atlanta, GA. E.E.U.U: www.atsdr.cdc.gov/es/toxfaqs/ es tfacts7.html.

8. Bansal M. Garg, U., Singh, V. \& Garg, V. (2009). Removal of $\mathrm{Cr}(\mathrm{VI})$ from aqueous solutions using pre-consumer processing agricultural waste: A case study of rice husk. Journal of Hazardous Matherials, 162 (1): 312-320.

9. Bello, G., Cid, R. \& Garcia y Arraigada, R. (2002). Carbon molecular sieves from Eucalyptus globulus charcoal. Microporous and Mesoporous Materials, 56 (2): 139-145.

10. Crini, G. (2006). Non-conventional low-cost adsorbents for dye removal: a review. Bioresource technology, 97 (9): 1061-1085.

11. Dean, J. \& Beverly, M. (1958). Extraction and Colorimetric Determination of Chromium with 1, 5-Diphenylcarbohydrazide. Analytic Chemistry, 30 (5): 977-979.

12. Doria, G., Hormaza, A. \& Gallego, D. (2011). Cascarilla de arroz: material alternativo y de bajo costo para el tratamiento de aguas contaminadas con Cromo (VI). Gestión y Ambiente, 14 (1): 73-84.

13. FEDEARROZ. (2006). Indice mensual de producción de Arroz. Revista de Arroz (27).

14. Gonzalez , M., Araujo, C., Pelizaro, C., Menesez, E., Lemos, S., Batista de Sousa, G.,et.al. (2008). Coconut coir as biosorbent for $\mathrm{Cr}(\mathrm{VI})$ removal from laboratory wastewater. Journal of Hazardous Materials, 159 (2-3): 252-256.

15. Gupta, V. \& Suhas. (2009). Application of low-cost adsorbents for dye removal a review. Journal of environmental management, 90 (8): 2313-2342.
16. Haiping, Y., Rong, Y., Hanping, C., Dong, H. \& Chuguang, Z. (2007). Characteristics of hemicellulose, ceIlulose and lignin pyrolysis. Fuel, 86 (12-13): 1781-1788.

17. Hormaza, A., Figueroa, D. \& Moreno, A. (2012). Evaluación de la remoción de un colorante azo sobre tuza de maíz mediante diseño estadístico. Revista de la Facultad de Ciencias, 1 (1): 61-71.

18. Juang, R., Tseng, R. \& Wu, F. (2001). Role of microporosity of activated carbons on their adsorption abilities for phenols and dyes. Adsorption, 7: 65-72.

19. Kishore, K., Krishani, X., Chiristodoulatos, C. \& Bobdu., V. (2008). Bioadsorption mechanism of nine different heavy metals onto biomatrix from rice husk. Journal Hazardous Materials, 153 (3): 1222-1234.

20. Kumar, U. \& Bandyopadhyay, M. (2006). Sorption of cadmium from aqueous solution using pretreated rice husk. Bioresource Technology, 97 (1): 104-109.

21. Marshall, W., Wartelle, L., Boler, D., Johns, M. \& Toles, C. (1999). Enhanced metal adsorption by soybean hulls modified with citric acid. Bioresource Technology, 69 (3): 263-268.

22. Mohamed, M.M. (2004). Acid dye removal: cComparison of surfactant-modified mesoporous FSM-16 with activated carbon derived from rice husk. Journal of CoIloid and Interface Science, 272 (1): 28-34.

23. Rodriguez- Reinoso, F. (1997). Activated carbon, structure, characterization, preparation and applications. In H. Marsh, E.A. Heintz \& F. Rodríguez-Reinoso (eds.). Introduction to Carbon Technologies. Alicante: Universidad de Alicante.

24. Singh, K., Hasan, S., Talat, M., Singh, V. \& Gangwar, S. (2009). Removal of $\mathrm{Cr}$ (VI) from aqueous solutions using wheat bran. Chemical Engineering Journal, 151(1-3): 113-121.

25. Tewari, N., Vasudevan, P. \& Guha, B. (2005). Study of $\mathrm{Cr}(\mathrm{VI})$ by Mucor hiemalis. Biochemistry Engineer Journal, 23 (2): 185-192.

26. Valverde, A., Sarria, B. \& Monteagudo, J. (2007). Análisis comparativo de las características fisicoquímicas de la cascarilla de arroz. Scientia et Technica, 37:255-260.

27. Wang Ngah, W.S. \& Hanafiah, M.A.K.M (2008). Removal of heavy metal ions from wastewater by chemically modified plant wastes as adsorbents: A review. Bioresource Technology, 99 (10): 3935-3948.

28. Wong, K.K., Lee, C.K., Low, K.S. \& Haron, M.J. (2003). Removal of $\mathrm{Cu}$ and $\mathrm{Pb}$ by tartaric acid modified rice husk from aqueous solutions. Chemosphere , 50 (1): 23-28.

29. Yang, H., Yang, R., Lee, D. \& Zheng, C. (2007). Characteristics of hemicellulose, cellulose and lignin pyrolysis. Fuel, 86 (12-13): 1781-1788. 